\title{
Úteros en alquiler *
}

\author{
JESÚS ZAMORA BONILLA \\ Universidad Carlos III de Madrid
}

\section{Introducción}

Un conocido aforismo de Karl Kraus dice: « ¿Quieres estudiar la ética de los negocios? Entonces deberás decidirte por hacer una cosa o la otra.» La sabiduría convencional sobre las relaciones entre ética y economía viene ciertamente afirmando lo mismo desde que existe la mismisima actividad mercantil: «la moral» tiene un ámbito propio (digamos, el de las relaciones personales) y ala economía otro (el de las relaciones comerciales), y ambos no se suelen mezclar, aunque, cuando lo hacen, es el primero el que más fácilmente cede ante el segundo, como cuando los personajes de El padrino aseguran, al mandar a un antiguo compañero a la muerte, que "no es nada personal, son sólo negocios».

Uno de los reductos fundamentales de la moral (así concebida desde el pensamiento económico) era el de las relaciones familiares, las cuales, si exceptuamos algunos trabajos de Gary Becker y sus seguidores, han sido consideradas por la mayor parte de los economistas como algo no interpretable mediante los conceptos económicos. La idea básica era que las relaciones familiares están tan fuertemente condicionadas por consideraciones puramente morales, que las decisiones internas de la familia no pueden ser analizadas a través del modelo del homo aconomicus, el individuo maximizador de su propio bienestar, habitante casi exclusivo de los modelos económicos. Las familias (o, como se dice en los manuales de macroeconomía, los «hogares»), y no los individuos, serían así las unidades elementales de aquellas decisiones sobre el consumo, la inversión, el ahorro, el trabajo, etc., que afectan al mercado, mientras que la distribución de los bienes y del trabajo dentro de la familia sólo podría explicarse por la aceptación e internalización de normas éticas y sociales. La teoría económica ha andado en esto (con la ya mencionada excepción) bastante a la zaga de otras actividades intelectuales, como la literatura y el cine, donde las unidades familiares aparecen muchas veces compuestas por verdaderos «maximizadores del bienestar individual», poco sensibles a consideraciones éticas.

El tema de este breve artículo no será, de todas formas, un análisis à la Becker del «mercado de úteros en alquiler». Mi objetivo cs solamente escudriñar algunas relaciones básicas entre la ética y la economía tomando como punto de partida el ejemplo de un tal "mercado», que no me atrevo a calificar como hipotético. Me plantearé preguntas como las siguientes: Len qué medida influirán las consideraciones morales en las decisiones que tienen lugar dentro de ese mercado y sobre ese mercado?; icuál debería ser el papel del Estado en su regulación?; ¿qué pueden decir sobre este asunto los criterios que se utilizan habitualmente en el estudio normativo de la economía (eficiencia de Pareto, funciones de bienestar social, etc.)? En definitiva, aprovecharé el tema de los «úteros en alquiler» como una excusa para reflexionar sobre ciertos aspectos éticos

* Este artículo se inscribe en el marco del proyecto de investigación PB 95-0125-C06 de la DGCYT, titulado «Ciencia y Valores». 
del sistema de mercado y sobre algunos de los principales enfoques normativos de la teoría económica ${ }^{\text {I. }}$.

\section{La perspectiva liberal}

En una transacción del «mercado de servicios reproductivos» que estamos considerando intervienen normalmente cuatro unidades fundamentales: en primer lugar, una pareja cuyos dos miembros producen gametos normales, pero que no puede satisfacer su deseo de tener descendencia porque la matriz de la mujer no existe o es incapaz de llevar a término el embarazo; en segundo lugar, una segunda mujer que se presta a que sean implantados en su útero uno o varios embriones de la pareja, y a ceder a ésta el bebé o los bebés que resulten de su posible gestación; en tercer lugar, una institución médica que se encarga de realizar la fecundación in vitro y la implantación de los embriones, así como de atender el embarazo y el parto; $y$ en cuarto y último lugar, el niño o los niños nacidos a consecuencia del proceso anterior. Por esta operación, la pareja paga una cierta cantidad de dinero, con una parte de la cual se retribuye a la institución médica y con otra a la «madre de alquiler».

Desde la perspectiva liberal, dominante en el pensamiento económico, la operación de «alquiler del útero» es un ejemplo típico de «mejora paretiana»: todas las partes involucradas en la transacción están mejor después de realizarla que antes (en términos económicos: aumenta su «bienestar» o «utilidad»), o al menos eso esperan, como lo demuestra el hecho de que todos participan en ella voluntariamente (en el caso del niño, hemos de hacer además el supuesto razonable de que es mejor vivir que no existir, al menos en términos de expectativas). Es también de suponer, para esta perspectiva, que ninguna persona ajena a dicha transacción mejorará o empeorará su situación por el hecho de que ésta se lleve o no a cabo. Por lo tanto, puesto que algunos individuos mejoran $y$ nadie empeora, la transacción debería efectuarse, 0 , por lo menos, no habría razones justificadas para prohibirla. Dicho de otra manera, la regulación pública de este mercado, sicmpre que restrinja el número de transacciones de esta clase o las elimine por completo, conducirá a un estado social ineficiente, en el que una wliberalización» produciría claras mejoras.

\section{Considerando las extemalidades}

El planteamiento del apartado anterior, que se limita a ejemplificar el principio normativo básico de la teoría económica liberal, divide a todos los agentes en dos categorias: la de aquellos que participan en una transacción y la de quienes no participan. El criterio de eficiencia de Pareto debe tener en cuenta, naturalmente, a los agentes incluidos en ambas categorias; es decir, hay que considerar la posible producción de externalidades (aquellos casos en los que las decisiones que toman los miembros de la primera categoría afectan al nivel de bienestar de los de la segunda). En otras palabras, hemos de examinar el supuesto de que nadie ajeno a la transacción mejora o empeora su situación por el hecho de que la misma se produzca. Ciertamente, la mera existencia de una polémica sobre la conveniencia o la moralidad del «alquiler de úteros» hace pensar que aquel supuesto no es tan fácilmente asumible como parecía. Olvidándonos do los casos en los que la situación de terceros puede mejorar (pensemos, por ejemplo, en los familiares y amigos de la pareja estéril —que probablemente se alegrarán del nacimiento-, o en los fabricantes de pañales que podrán vender más-), una lista de posibles externalidades negativas incluiría, entre otros, a los siguientes sujetos: 
a) personas que rechazan la «transacción» -y, por lo tanto, ven reducida su «utilidad»-por considerar inmoral la manipulación artificial de la reproducción humana;

b) personas que ven reducida su «utilidad» porque, no teniendo los recursos necesarios para realizar una transacción de ese tipo, y deseando efectuarla, preferirian que ninguna otra pareja pudiera hacerlo, especialmente si ésta lo consigue gracias a su mayor poder económico;

c) personas a las que va a perjudicar por unas u otras razones la gestación o el nacimiento de los niños nacidos asi (por ejemplo, los otros hijos de la «madre de alquiler», si ésta fallece o se ve gravemente afectada a causa del nuevo embarazo, 0 los comerciantes que se vean afectados negativamente por el cambio en los hábitos de consumo de la pareja).

El supuesto de ausencia de externalidades negativas es, por lo tanto, muy poco realista. En un caso como el que estamos considerando, es prácticamente seguro que habrá algunas personas que resultarán perjudicadas si la transacción se lleva a cabo, aunque las razones por las que su utilidad vaya a disminuir, y la medida en la que lo haga serán muy diferentes en cada caso y tendrán implicaciones morales también diferentes (nos referiremos a esto más adelante). Podemos concluir entonces que es inapropiada la aplicación ingenua del criterio de Pareto al mercado de úteros en alquiler, tal como la formulamos en el apartado precedente. La mera «liberalización» de ese mercado, en el sentido de permitir participar en él a todos los que lo desearan y pudieran pagar el coste de tales servicios, no supondrá necesariamente una "mejora paretiana», y su justificación ética deberá hacerse mediante otros criterios, varios de los cuales examinamos a continuación.

\section{Algunas altemativas}

El principio de Pareto, entendido como criterio básico para la evaluación moral de las distintas regulaciones económicas, es muy insuficiente en el sentido de que resulta inaplicable a la mayoría de los casos en los que debe tomarse alguna decisión como la que estamos estudiando en este artículo, es decir, decisiones sobre cómo deben llevarse a cabo (o si deben en absoluto realizarse) la producción y la distribución de ciertos tipos de bienes o servicios. Ésta es una limitación bien conocida de la «nueva economía del bienestar», uno de cuyos axiomas fundamentales era la imposibilidad de efectuar comparaciones intersubjetivas de utilidad. Este axioma fue aceptado sobre todo por motivos de carácter epistemológico: las afirmaciones sobre si el bienestar de una persona aumentaba o disminuía más que el de otra, $e$ incluso si era mayor o menor que el de la otra en têrminos absolutos, no se consideraban susceptibles de ser contrastadas por medios empíricos, y, por lo tanto, eran tachadas como sacientíficas». El único criterio moral compatible con la «cientificidad s de la teoría económica era el de Pareto: un cambio de situación es aceptable si el bienestar de algunas personas aumenta y no disminuye el de ninguna. Ahora bien, si el "cambio de situación» que se está considerando se reficre a si una cierta clase de actividades económicas debe ser permitida o no, entonces es muy probable que todas las altemativas factibles (incluido el propio statu quo) conduzcan al empeoramiento del bienestar de algunos individuos, con lo cual el criterio de Pareto no nos ofrecerá ninguna respuesta sobre qué alternativa es la preferible. Peor todavía: si el principio de Pareto se interpreta, como algunas veces se hace, en el sentido de que kprohibe» aquellos cambios que empcoran la situación de algún agente, entonces casi nunca se consideraría aceptable ningún alejamiento del statu 
quo. Ni que decir tiene que esta interpretación es la preferida por el pensamiento conservador.

Si nos sentimos insatisfechos con el principio de Pareto, contamos con varios enfoques alternativos, probablemente más fecundos a la hora de generar juicios de valor sobre el ordenamiento económico. Estos enfoques podemos agruparlos en tres grandes categorías:

1) Aquellas que fundamentan los juicios normativos en la agregación de las funciones de bienestar individuales; dentro de este enfoque tenemos, en general, la llamada "teoría de la elección social», y particularmente el utilitarismo ${ }^{2}$.

2) Las que basan los juicios norma. tivos en el acuerdo de los individuos sobre las nomas que van a regir sus relaciones; esta categoría incluye fundamentalmente a la llamada "economía política constitucional», $y$, en general, a las concepciones contractualistas ${ }^{3}$.

3) Aquellas que fundamentan los juicios normativos en la preexistencia de ciertos derechos poseídos por los individuos; esta categoría incluye muy diversos enfoques, desde los más liberales, que toman como derechos básicos el de la propiedad privada y el de la libertad económica, hasta, los enfoques más izquierdistas, que toman como derecho más elemental el de la igualdad en la satisfacción de las necesidades primarias ${ }^{4}$.

En los siguientes apartados examinaré el mercado de «úteros en alquiler» desde la perspectiva de cada una de estas tres categorias, aunque lo que diré sobre ellas no será aplicable a todos los enfoques comprendidos bajo cada una, sino sólo a los que consideraré aquí (en una selección ciertamente discutible) como representativos de las mismas.

\section{Ei enfoque utilitarista}

Según el utilitarismo, en su versión estándar, la evaluación de las decisiones y las reglas económicas debe llevarse a cabo considerando una efunción de bienestar social" definida por la suma del bienestar de cada miembro de la sociedad. La principal conclusión que podemos sacar a partir de aqui es que el paso de una situación a otra será aceptable moralmente si (y sólo si) el incremento del bienestar de los que resultan beneficiados por dicho paso es mayor que la disminución de bienestar que sufren los perjudicados. Evidentemente, la cuantificación del bienestar de cada individuo no puede hacerse de forma totalmente objetiva, pero los defensores del utilitarismo argumentan que nuestra familiaridad con las circunstancias de la vida humana nos permite hacer en muchos casos «comparaciones intersubjetivas de utilidad (o de bienestar) $\gg$ plenas de sentido y aceptables sin problemas por casi todo el mundo.

En el caso que estarnos tratando, la cuestión es, entonces, la de si el incremento en el bienestar (efectivo o estimado) de los agentes que intervienen en la transacción, junto con el de aquellos beneficiados por externalidades positivas, compensa a la pérdida de bienestar (efectiva o estimada) de quienes pueden verse perjudicados por ella. Nuestro conocimiento «de sentido común" sobre las funciones de bienestar de nuestros conciudadanos es, desgraciadamente, muy poco útil para decirnos si la alegría de unas cuantas docenas de parejas por su difícil y deseada paternidad, junto con la satisfacción de las otras personas que parlicipan en las operaciones, y el bienestar que alcanzarán los niños nacidos gracias a ellas, supera o no a los posibles perjuicios y a los sentimientos de indignación, envidia o repulsa que otros muchos experimentarán. Es muy probable que la valoración que hagamos del mercado de úteros en alquiler, incluso 
basándonos en una perspectiva utilitarista, otorgue un peso diferente al bienestar de cada individuo involucrado, según la importancia o el valor moral que concedamos a cada tipo de "satisfacción» y de «perjuicio" (por ejemplo, según a qué gnupo pertenezean de los definidos en el apartado 3); pero esto nos aleja de la perspectiva utilitarista, pues ésta no nos ofrece criterios sobre qué «satisfacciones» y «perjuicios» son más o menos importantes.

\section{El enfoque constitucional}

Desde la perspectiva constitucionalista, una norma es aceptable si pudiese haber sido aceptada de forma unánime en una asamblea constituyente en la que participaran (directa o indirectamente) todos los miembros de la sociedad, los cuales (justo por tratarse de una asamblea constituyente, en la que se van a aprobar normas muy generales, y que tendrán vigencia durante un periodo de tiempo muy largo) ignorarían en gran medida cómo van a verse afectados por esa norma a título individual. Dicho en términos clásicos, las normas constitucionales aceptables serían las que podría aprobar una asamblea cuyos miembros estuvieran sometidos al «velo de la ignorancia» $\alpha$ «de la incertidumbre». En estas circunstancias, los votantes tenderán a elegir (o al menos así es de suponer) las normas que, por una parte, más beneficien al «individuo medion, es decir, a aquel individuo con el que más probablemente se identificarán al despojarse del "velo», y que, por otra parte, produzcan un nivel de bienestar mínimamente aceptable para todos los individuos.

Aunque diversas escuelas han sacado conclusiones políticas diferentes a partir de este enfoque, por lo general todas ellas tienden a aceptar que la constitución aprobada en tales circunstancias de incertidumbre establecerá la existencia de un Es- tado en mayor o menor medida redistribuidor (cómo controlar a su vez a dicho Estado en su «afán recaudador» es el otro gran problema de la economía constitucional). Es decir, los individuos aceptarian restringir parcialmente sus libertades económicas a cambio de un cierto margen de «seguridad». Llevado al tema de nuestro artículo, podríamos interpretar esto $\mathrm{cn}$ el sentido de que, si los constituyentes ideales no saben cuál va a ser su status económico, pero consideran deseable la posibilidad de tener descendencia mediante el «alquiler de ưteros», tal vez decidan no dejar estas operaciones en manos del libre mercado, sino ofrecerlas de modo general a toda la población como una prestación más de la seguridad social. Esto resolvería la cuestión relativa al segundo grupo de posibles perjudicados considerado en el apartado 3. Con respecto al tercer grupo, es probable que la incertidumbre sobre la distribución de ventajas y desventajas sea tan alta, frente a la certidumbre en los bencficios obtenidos por los participantes en la transacción, que ésta se considere accp. table de todas formas.

Ahora bien, respecto al primer grupo de «perjudicados», una dificultad del enfoque constitucional es que no nos dice si, tras el "velo de la ignorancia", los individuos olvidan sus valores morales además de su status económico. Si esos valores no se «olvidan» (como es de suponer en una asàmblea constitucional mínimamente realista), es entonces posible que quienes consideran el alquiler de úteros como algo rechazable moralmente voten en contra de su existencia, independientemente de cuáles pudieran ser sus expectativas como posibles «usuarios» del mercado de servicios reproductivos, e impidiendo de esta forma la obtención de la necesaria unanimidad que requiere, scgún este enfoque, la elección de una norma de carácter constitucional. 


\section{El enfoque de los derechos}

Para las dos perspectivas que acabamos de examinar, los derechos de los individuos no son previos al mecanismo fundamental de toma de decisiones postulado por cada una, sino que más bien esos derechos sólo existen como resultado de la aplicación de tales mecanismos. Asî, para el utilitarismo, los derechos de los individuos serian simplemente aquellos que permiten maximizar el bienestar agregado, y para la perspectiva constitucional, los derechos serian aquellos que aprobara de forma unánime una asamblea constituyente. Pues bien, una crítica que podemos hacer a estas dos perspectivas es que, al intentar definir los derechos sobre la base de algún criterio anterior a los mismos, están cometiendo una petición de principio, pues la aplicación de estos criterios da por asumidos ya unos ciertos derechos: en el caso del utilitarismo, el derecho de cada persona (pero no de los otros seres vivos, por ejemplo) a que su nivel de bicnestar sea tenido en cuenta dentro de la función de bienestar social (digamos el «derecho a ser tenido en cuenta»), así como el derecho de que el bienestar de cada individuo reciba el mismo peso que el de los demás en dicha función (digamos el aderecho a la imparcialidad»); en cl caso del segundo enfoque, el derecho a participar en la asamblea constituyente, a que el voto de cada uno cuente lo mismo que el de los demás, y, además, cl derecho a que el voto exprese realmente la propia elección óptima de cada uno (para lo cual los individuos deben recibir la información adecuada, deben estar libres de coacciones externas, etcétera).

En realidad, tanto el enfoque agregativo como el contractualista pueden ser considerados como dos casos especiales del enfoque de los derechos, en el sentido de que ambos parten de la presuposición de ciertos derechos fundamentales de los individuos, e intentan sacar las consecuen- cias normativas que se deducen de ellos. La diferencia con otros enfoques estribaria en que los derechos fundamentales se intentan reducir al mínimo mediante el recurso a considerar sólo aquellos que son necesarios para definir un mecanismo de generación de los otros derechos. El asunto está particularmente claro en el enfoque contractualista o constitucional: según este enfoque, el fundamento do todo ordenamiento normativo está en la capacidad que tienen los seres humanos para pactar con sus semejantes. En el utilitarismo, en cambio, no estâ muy claro por quể se debería tener en cuenta sólo (o en mayor medida) la utilidad de los seres humanos en la función de bienestar «social»; es difícil imaginar una respuesta a esta pregunta que se pueda basar en argumentos puramente utilitaristas.

Otros enfoques de los que consideramos en este apartado incluyen, simplemente, más dercchos (y deberes) fundamentales que el simple derecho a ser tenido en cuenta en igualdad de condiciones. Naturalmente, es aquí donde nos encontramos con las principales dificultades al abordar nuestro tema: bes un derecho fundamental el de buscar descendencia por cualquier medio médicamente posible (en cuyo caso el "alquiler de úteros" podría ser razonablemente una prestación más de la seguridad social)?, 60 el de disponer libremente de los propios órganos sexuales para ayudar a la reproducción de terceros?, io el de llevar a cabo las transacciones que uno desee con los recursos de los que legitimamentc disponga (con lo cual el mercado de úteros en alquiler sería un mercado libre, igual que los demás, y limitado a quienes pueden pagar el precio de dichos servicios)? La respuesta a todas estas cuestiones dependerá, sin duda, de la concepción de la persona que tome como punto de partida cada uno de los enfoques normativos que se ocupen de nuestro problema. Por ejemplo, desde un planteamiento feminista puede conside- 
rarse que la venta de la capacidad reproductiva conducirá probablemente a una nueva forma de explotación de la mujer (en definitiva, serán las mujeres con menos recursos las que se vean abocadas a ofrecer este tipo de servicios) ${ }^{5}$. Desde un planteamiento liberal, lo importante es la capacidad que tenga cada uno para aprovechar las oportunidades que le ofrece el mercado, capacidad que nadie tiene derecho a limitar. Desde una perspectiva religiosa, la transformación de la función reproductiva en una mercancía supondría una lesión inaceptable de la dignidad sagrada de la persona.

Evidentemente, las valoraciones normativas que se efectúen desde cada enfoque no deben ser totalmente apriorísticas; con esto quiero decir que las consecuencias económicas y de otro tipo que tendría el definir y garantizar unos ciertos derechos han de ser tenidas en cuenta, pues tal vez constituyan situaciones indeseables desde el punto de vista de esos mismos derechos. Salvo para aquellos enfoques que afirmarían algo así como fiat justitia et pereat mundus, una cierta dosis de razonamiento económico es tan conveniente como una cierta dosis de sentido común (si es que, en muchos casos, no son la misma cosa). Por ejemplo, los enfoques que consideran moralmente correcto el alquiler de úteros, tal como lo estamos considerando aqui, tal vez no vean con tan buenos ojos el que una mujer se preste a concebir un hijo cuya paternidad ceda después a la pareja estéril a cambio de una cantidad de dinero que puede ser incluso menor que en el primer caso (al fin y al cabo, aquí corre menos riesgos de tipo médico). En cambio, quienes critican la prestación de servicios reproductivos por motivos de tipo religioso, tal vez sí que considerasen aceptable (en el caso de que fucra médicamente posible) que el embrión de una moribunda fuera traspasado al de otra mujer que pudiera llevar el embarazo a término, incluso aunque ésta sólo estuviera dispuesta a hacerlo a cambio de una cierta cantidad de dinero, tal vez $\mathrm{ma}$ yor que la que exigiría en el caso de «alquilar su uterom en condiciones menos traumáticas.

\section{Algunas consecuencias}

La principal consecuencia que podemos sacar de la discusión anterior es negativa: los instrumentos de la teoría económica normativa son de poca utilidad a la hora de resolver los dilemas morales del tipo que estamos estudiando, es decir, dilemas sobre si una cierta clase de transacciones económicas debe ser autorizada, regulada o prohibida. Algunos criterios, como el de Pare to o cl utilitarista, no nos dan ninguna respuesta a la cuestión, mientras que otros, los basados en los derechos, nos dan tal vez demasiadas respuestas y pocos criterios efectivos para clcgir cntre cllas.

Ahora bien, esta dificultad no debe llevarnos a una conclusión todavía peor: la de que, puesto que la ética parece ser incapaz de dar una respuesta clara sobre el ordenamiento normativo de la economía, habría que dejar que el sistema económico se desarrolle alibre de restricciones éticas». Tal conclusión es simplemente una contradicción en los términos, pues ella misma es una afirmación moral (pensar en el «habría que»). En realidad, el sistema económico es la suma y la interacción de muchísimas decisiones individuales y colectivas, $y$ en estas decisiones siempre tienen un peso, mayor o menor, según los casos, las consideraciones éticas (por ejemplo, es indudable que la moral intervendría con mucha más fuerza en la decisión sobre alquilar el propio útero o contratar tales servicios que en la decisión sobre si comprar una marca u otra de automóviles). Entre el deseo de obtener la mayor niqueza posible y el deseo de expresar, fomentar y vivir de acuerdo con los propios valores, existe una inevitable interde- 
pendencia (no siempre negativa), que los economistas harían bien en estudiar te6rica y empíricamente, con el fin de comprender mejor el propio funcionamiento del sistema económico y las consecuencias que podemos esperar de nuestras propias decisiones ${ }^{6}$. Posiblemente el camino más interesante para este tipo de estudios es el abierto por la «nueva economía insti- tucional», en la que las normas morales $\mathrm{y}$ sociales, que sirven de base al proceso económico a la vez que lo conforman y (en muchas ocasiones) lo dificultan, son objeto de análisis explícito, pero no tomadas necesariamente en un sentido anormativo", sino más bien como elemento fundamental de la realidad que se pretende estudiar?

\section{NOTAS}

- Una excelente introducción en castellano a los criterios normativos fundamentales de la economia de] bienestar y de algunos enfoques alternativos es la de A. Casahuga, Fundamentos nomativos de la accion y organización social, Aricl, Barcelona, 1985. En inglés puede verse K. W. Rothschild, Ethics and Economic Theory, Edward Elgar, Aldershot, 1993.

2 Pueden verse A. Sen, Elección colectiva y bienestar saciah, Alianza Editorial, Madrid, 1976, y S. Barberá, «Desarrollos recientes en la teoria de la elección socialn, Hacienda Pública Espaniola, núm. 44, 1977, pp. 247-269. Un resumen màs reciente lo ofrece $A$. Villar, «La lógica de la elección social: una revisión de los resultados bảsicos", Investigaciones Económicas, vol. XII, núm. 1, 1988, pp. 3-44. El utilitarismo ha sido defendido especialmente por J. C. Harsany, a partir de su "Cardinal Welfare, Individualistic Ethics and Interpersunal Conparisons of Utility, Joumal of Political Economy, núm. 63, 1955, pp. 309-321; véase tanbién A. Sen y B. Williams (eds.), Utilitarianism and Reyond, Cambridge University Press, Cambridge, 1982.

* Véanse, por ejemplo, J. Buchanan y G. Tullock, The Calcalus of Consent, University of Michigan Press, Ann Arbor, 1962 (trad. castellana en Espasa-Calpe); G. Brennan y J. Buchanan, The Reason of Rules, Cambridge University Press, Cambridge, 1985 (trad. castellana en Unión Editorial); J. Rawls, $A$ Theory of Justice, Harvard University Press, Cambridge, Mass., 1971 (trad. castellana en Fondo de Cultura Económica), o V. J. Vanberg, Rules and Choice in Economics, Routledge, London, 1994.

"En el enfoque «de derechas", véase R. Nozick, Anarchy, State and Utopia, Basic Books, New York, 1974 (trad. castellana en Fondo de Cultura Economica). En el enfoque wde izquierdas", véanse A. Sen, On Eihics and Economics, Basil Blackwell, Oxford, 1987 (trad. castellana en Alianza Editorial) y P. van Parijs, Real Fredom for All: What (if Anything) Can Justify Capilalsm?, Oxford University Press, Oxford, 1995 (trad. castellana en Paidos).

${ }^{3}$ Desde otros enfoques feministas se puede pensar, en cambio, que la posibilidad de kalquilar el útero» implicará un mayor control de la mujer sobre sus capacidades reproductivas, $y$, por lo tanto, una mayor libertad. Una discusión semejante sobre la «mercantilización del sexon entre varios enfoques feministas se aborda en E. Anderson, Value in Ethics and Economics, Harvard University Press, Cambridge, Mass., 1993, pp. 150 y ss.

" Mc limito aquí a repetir la opiniôn de A. Sen en su On Ethics and Economics, cit.

7 Véase, por ejemplo, D. C. North, Institutions, Institutional Change and Economic Performance, Cambridge University Press, Cambridge, 1990. 